\title{
We Can Think with the Implicit, As Well As with Fully-Formed Concepts
}

[p. 147]*

\author{
Eugene T. Gendlin
}

We can now adopt a new understanding of scientific knowledge and its role in our society. The concepts which science presents change every year. Neither today's nor next year's concepts are representations of reality. Many people mistrust science altogether and gladly adopt anything from any other source. Our best thinkers also attest to the fact that every picture, every representation, every theory and set of concepts can break down and be found false.

On the other hand it has become quite impossible to live without science. Science has already gone into most of the things we touch all day. Without science six billion people could not live in our crowded space.

Neither believing nor attacking the scientific pictures makes sense. Every scientist is aware of the constant change and ambiguity in every field, but no critic of science would like to board an untested airplane or do without electricity and computers. The scientific concepts are not just true but they are not just invented.

We cannot get further if we stay within concepts. But we can shift from the concepts to consider how they are generated. Instead of being trapped in the picture of nature which science presents, we can think about the process by which concepts are constantly formed and reformed in a wider context. We can examine the reciprocal interaction, the zig-zag between the wider context and the changing conceptual pictures.

To think about concept-formation is most urgent where technology is applied to human beings. This is studied so poorly and primitively, there is no real science of applications at all. Compare the market-application studies with the process in the natural sciences where every new finding is replicated in many laboratories. Every study is many times improved upon. The instruments and measures are based on many layers of careful studies. In contrast, where technology is applied to millions of people, what masquerades for "science" consists of one or two studies never actually replicated, always on the starting level without validated measures, often paid for by parties interested in the market. The well-earned respect for science is mistakenly transferred to these few studies. Government policy

committees feel forced to heed such "findings." Where technology is applied to life there is urgent need to become able to think beyond "science." (Gendlin, 1997b, Footnote 18)

In every kind of knowledge we can look at the process, the activity by which the knowledge is generated. Here lies a whole territory that has always been treated poorly. Traditional philosophy of science told a simplistic story which no working

\footnotetext{
* Page numbers refer to the pages of the article as contained in the book After Cognitivism (2009; K. Leidlmair, ed.;
} Springer). 
scientist could use. Actual observations of scientific activity are rare. How science is generated in practice is left mostly to a kind of political process among scientific institutes, journal editors, and grant-giving agencies. On the theoretical side, the process of concept-formation has been left almost entirely dark. New concepts seem to come to scientists in the shower or in dreams, at any rate in their private space.

A large scientific project involves many kinds of people with different functions. There are laboratory people, equipment designers, many kinds of specialists. Usually there is one theorist in the whole project. When the findings are surprising, this theorist goes home to revise the theory, while everyone else waits. The theorist returns next morning or a few days later with the best possible revision that can be made to bring the theory just a little closer to the findings. He also brings questions which lead everyone to discuss and reexamine the equipment and all the procedures and circumstances. Each kind of specialist works in a different context, including the designers of the equipment and the graduate students who run the labs. They all reenter their implicit contexts and then some of them will have something to say. The theorist goes home and returns a few more times (see also Crease, 2004).

Why can only the special theorist revise the theory? It is because the theory is embedded in a large context in which it arose. It was fashioned to take account of many considerations. Some changes in it have already been proposed over the years, and for various reasons. Every concept in the theory is embedded in detail, some defined, some anecdotal. Any revision will force changes in related theories. Revising a theory would be easy if one could simply change it to fit new findings. But the revision has to fit everything else too! Every concept is logically connected to other relevant concepts. All this far exceeds what can be thought bit by bit, one bit at a time. It requires feeling the whole context at once, so that precise logic can be used in a relevant way. This is possible only for someone who is spending years consistently living and working with that theory. Only that individual can hope to come up with a workable revision.

Many factors will be fed into computers with various models. But the whole context does not consist of definable units such as a computer requires (Gendlin, 1997b). Revising a theory is precisely what computers cannot do. This fact can lead to a central recognition:

Dreyfus has written pioneering works about the fact that computers cannot understand or create metaphors. Metaphor does not reduce to a set of rearranged parts. Language is not just a system of tags for separate things. Languaging consists of newly modulated meanings. Much of what we say is repetitious, but several times a day we find ourselves in unusual situations without a routine. Odd sentences come

[p. 149]

to us. The old words come in new phrases with new meanings. No computer can create such sentences, nor can a computer respond sensibly to them. But a person can. The human process does not remain within a set of unit meanings. It involves an implicit context which must be had as such.

In metaphors, in fresh word-use, and in revising a theory something functions 
implicitly beyond the defined units. In every creative process something implicit exceeds the discrete units. This happens wherever we look for novelty and change.

For example, psychotherapy. My philosophy led to quantitative research to pinpoint a variable that correlates with successful outcomes when most other measures do not. Successful clients much more often refer to something implicit that is palpably sensed and spoken from. They use a characteristic mode of language which can be reliably recognized on tape recordings.

We went on to create a step by step training system for direct reference to the implicit. There is now a world-wide network of trainers and users. (See www.focusing.org)

In recent decades a major social change is noticeable: Many more people have become able to refer directly to the implicit, or can learn to do so. But there are great differences among people. Some refer to the implicit only in very odd situations; others do it frequently all day, giving them much greater capacities with most situations. The degree of depth also varies. Some people can directly refer to a bodily-sensed implicit meaning; some find it by going back into where they have just spoken from.

Language is part of the human body's interaction in situations. Language-forms and civilized human situations developed together and are sensed implicitly in the body. (See Gendlin, 1991, 1995) When we think freshly into something that is not yet clear to us, fresh phrases come to us.

We have also devised a step by step procedure for fresh thinking which has been used by high level theorists and by eighth graders. (See Gendlin, 2004) We ask people to work on something they deeply know but have been unable to say much about. We ask them to write a few sentences and underline key words. Then we ask them, quietly and invitingly: "If this word could mean just what you want it to mean, what would it mean?” In response come fresh metaphorical sentences to say what has never been said before.

If the new sentences still employ the usual "big words," the instructions are again: "If this key word could mean just what you want it to mean, what would it mean?" Fresh phrases need to replace all big words. To do this turns out to be possible both for ordinary people and for those who want to go on to create new concepts. For the latter we provide further steps to define precise terms. Fresh phrasings contain the roots of new concepts.

Fresh metaphorical language has the power to originate new meanings. From these one can define new concepts.

Language has been studied mostly as a system of words and grammar. The process of word-use has hardly been examined at all. Wittgenstein pointed out that no concepts determine what words mean. Concepts come later. The meanings of words

[p. 150] depend on their use in situations. We have gone on from him to study how words actually come to us. No one else has looked at how words come, so far as I know. If we enter into how they come, we can examine their implicit meaning directly. The implicit meaning is never equivalent just to the words, although it is what we mean by saying them. The words mean the change that saying them makes in a situation. Words do not 
represent; they do something. They mean what they do.

It turns out that all word-use is metaphorical in a new situation. To put it more precisely, metaphor and ordinary word-use are both instances of a wider process of "crossing" which opens a whole arena of philosophical questions that can now be worked on.

Philosophy has long been stymied before the problem that what we think about concept-formation is just a concept, not its making. No mere concept can replace the role of the person doing the concept-making. But why omit us? It is not as if we had to plan on people disappearing. We were taught that a concept should stand alone, be true alone, a representation.

A number of philosophers have advanced beyond the representational view. Wittgenstein did, although he said he could not say, only "show." Heidegger did in Being and Time, but then spent the rest of his life with the conundrum that what exists hides behind what it presents. Bakhtin and Bataille said that we can think beyond what is presented, but only in very odd cases. This has changed.

We need no longer be trapped within concepts cut off from their genesis and regenesis. There is no longer the problem of having only concepts. We can conceptualize so as to keep a concept connected to where it arises. We can re-enter there, and return with something further. The concept embodies its own capacity to be "revised."

Many new strategies of thought become possible. We can enter into the implicit context when we just used a concept, to find just what strand of its meaning was at work.

From the new precision we can generate a new set of units for logic and the computer. We need not always stay within our starting set of terms (Gendlin, 2004).

The new precision (which we then say) "was" implicit was not there as such before. Finding and formulating it may shift our understanding of the whole context. But the shift will not be to something different, rather to what (we then say) was what we really meant all along.

When people explicate something implicit they usually say that their words "match" their experience, as if they were comparing two forms. But an implicit sense does not have the kind of form that could match words or concepts. What people call "matching" is indeed an important relation between implicit and explicit but the relation is not representation. It is rather the characteristic continuity we experience when new sentences and then new concepts articulate and explain what we had understood only implicitly. We call this relation "carrying forward."

(A metaphor is one instance of carrying forward. See long derivations of this concept in Gendlin, 1991, 1992, 1995, 1997a, b).

The philosophical treatment of carrying forward is unavoidably complex because there is no such thing as an implying alone. Something implicit is always also

an explicit occurring. Direct reference is already a kind of symbolization, when we say "this," or "that whole thing," or even when we only point our attention. And even without attention, the events always (as it were) "symbolize" just this implying, and no other. There is always an inseparable implying-occurring pair, but a kind of separation is possible at our next move, because from the implying 
we can go on differently than we can from what occurred. We can go from any pair to a direct reference pair and from this to many other kinds of pairs. Each makes for a characteristically different kind of "logic." Seven major kinds of pairs have been examined. Or, we can move by logical inferences and from computer to computer on the explicit side. We can make long chains of either kind, or go between them (Gendlin, 1997c).

In our further move the implicit always responds with exactly this, always just so. The implicit is always highly demanding and leads to special phrases and concepts which can arise without any scheme. If a scheme is applied it crosses with the implicit to yield a specific result which could not have been found from the scheme alone. If one is just playing, there are many possibilities. If one is working on a problem, even trying many schemes may fail to produce an advance. But there is no arbitrary variety. From the implicit the various new concepts constitute a grouping, a "fan" that retains its link to its origin. We can think directly with implicit and fan. In this way we can employ many models, not just one. Each may lift out something relevant. We can also find what precise strand of a model functioned to do so. To "find" and define what functioned is always a further carrying forward.

We refuse to read a philosophical scheme into the implicit. The implicit is more intricate, more finely ordered than any scheme, as we see from its capacity to respond in a specific way to mutually exclusive schemes. I called the implicit "multischematic.” It does not consist of discrete units: I called it "nonnumerical.” It functions as an unseparated multiplicity.

Past present and future are not separate positions. All the past functions in new ways in the present. Everything that happens crosses with everything that happened. The implying of a next event is always a finely webbed intricacy. Top-down distinctions so often have no effect, no traction at all, whereas distinctions found from direct reference carry forward in their very forming and coming. From direct reference the words come to us already crossed in new phrases, crossed with everything that has led up to the present moment in that situation.

"Crossing," "carrying forward," and "unseparated multiplicity" are instances of a new kind of pattern. Patterns that emerge from explicating can seem "illogical" because they include their own relation to their implicit source. The relation cannot be represented before us. With such patterns we can generate logically connected stable concepts but they do not reduce to separable parts because they carry implying forward.

We do not lose the power of "flat" conceptual patterns if we study anything with new concepts that have the carrying forward power as well, and as philosophers we want especially to study the explicating process. We study it with phrases and concepts that retain their link to the process they explicate and instance.

[p. 152]

\section{Comparing the Two Kinds of Patterns}

When existence is thought of as filling space-time, our concepts are not understood as occurrences 
within the wider context of interaction, only as "about" entirely separate external things. Therefore they are taken as "representations." Truth is understood as representation, correspondence. Discrete external entities are understood in a positional patterning presented before us. Perception is taken as basic, and percepts are treated as if they were independent entities.

To consider a living process we cannot begin with perception; we must put interaction first. Thinking and research are living activities. We are always already in a contextual interaction with what we then conceptualize and re-conceptualize. Presentations relate to each other not only externally but also in carrying forward the interaction with them in which we live, operate, and understand them.

Although we can have bridges to the older kind of concepts, the new kind of patterns render things very differently than the current kind of concept. For example, the word "values" that facts exist, so that there is something separate called "values" which have to be brought to facts. The word "consciousness" assumes that human behavior and perception can be understood scientifically as spatial occurrences so that "consciousness" is something separate which has to be added and could be unnecessary in science.

We do now also have ecology and other holistic approaches. With our new approach living process can be understood as a sequence of carrying forward wholes.

Almost all common phrases and scientific concepts are still structured so as to render everything as something that exists in empty space and has separable parts. Earlier philosophers saw no way out. To get past this we replace the main old words with new metaphorical phrases and we formulate our new logically precise terms directly from them.

In experiments and applications the living things and people are assumed to be nothing but what the scientific concepts render. There is assumed to be nothing else sitting there. With the new concepts one would be able to think about the wider context in which the reductive entities are a changing subset.

Discrete entities with separable parts cannot be alive. Discrete entities have only external (formal, logical) relations to each other. In contrast, in living tissue, animal behavior and language the factors are crossed. From fresh metaphorical sentences we can define a new kind of terms that remain consistent and can employ logic, yet also have internal relations to each other. Because the terms relate to each other in both ways, the result of a logical inference can open major further understandings.

For discrete entities "existence” means filling space-time. Each “is.” In living process "existence" has a more intricate meaning. One finds not just discrete "is-entities, but always also a further implying. Occurring which is always also an implying is found in any topic that involves living, including the philosophical explicating of the living process of explicating, as we are just now doing. A living process never has only a static “is,” always also a further implying.

[p. 153]

Now we can sense that a great many things in our world could be understood very differently if we had concepts that include implying. A system of such concepts has been formed for a new understanding of living tissue, animal behavior and perception, and human symboling. (These concepts can also constitute bridges to the usual kind of concepts). Better ones will no doubt be devised once many people see understand this initial set of concepts. (A Process Model, www.focusing.org/process.html).

A living body is an environmental interaction. Body life is always also environmental eventing. 
A living body always implies its next whole occurring, and enacts it when possible. We can think about living tissue as coordinated interaffecting in which body-environmental sub-processes differentiate and imply each other.

Animals behave in a context of behavior possibilities. The enacting of any one behavior changes whether and how the others can occur.

Animals do not just move. Only humans have a perception of "just" moving, i.e., only a change of location in an empty space consisting of location points. This is a purely human symbolic creation which does not exist alone, but only with a bodily carrying forward to which one pays no attention. Even the highest monkeys cannot put two sticks together to make one long one, to reach some bananas. They cannot see a length just as a pattern outside themselves. They lack the capacity called "the external tie," to see things as if separate from themselves. This is the capacity of "homo faber," the power to make things by treating things as patterns that can be moved while nothing else changes, dividing and combining discrete parts, as if things were just spatial patterns.

Our new philosophical terms enable us to derive knowledge as representation and to show that the supposedly empty space is symbolic and rests on a more original bodily process. In a puzzling way it has been known that the presentations before us cannot represent existence.

Logic is powerful but of course it requires discrete units which are artificial products. Scientific prediction succeeds to the extent that we get the same result from the same operation under the same conditions. But the question is how a repeatable procedure is discovered, and a result becomes recognizably "the same." This is achieved only after a long time in which one gets nothing the same. And, when something does repeat, one may not know just what one did. Everyone in science knows this daily work and play.

So it is obvious that whatever we study does not come in already-cut units. In Austin's phrase, "there are no handy denotative packages" which can just be filled into logical relations and fed into a computer.

But this raises a vital question: If the changing presentations before us do not represent existence, why do they carry the implicit forward? Why do they further explain what we knew implicitly? What does "explain" mean here?

Logical patterns and patterned discretes "explain" by generating before us a whole field in which we can act in new ways and move and make new things. But this new moving and making also carries forward the living tissues and the context of behavioral possibilities in which we humans do not only move patterns, but of

[p. 154] course also eat and sleep and procreate. The usual kind of concepts cut the separate entities off from the wider process, as if they represented reality just because they explain and let us do and make so much. And of course, the new doing eventually leads to new findings which force changes in next year's presentations. So we need not complain about the changes either, but we can think with a new kind of concepts which incorporate and remain connected to the implicit context from which they arise.

When we make concepts from and with direct reference to the implicit, a new world opens. The doubled kind of patterns also generate a different kind of space in which we can move and act and understand in new ways. We are only at the very beginning of creating that new world.

When there will be a great many more such concepts, they will be a new kind of world in which we can live major parts of our lives. The social institutions (including science) will have changed. 
No longer will they employ so little of what a person can be. Currently our society seems to know nothing about what it is to be a person inside. But it can become understood that the audible language is only the top of a continuity with our intricate aliveness. People will be able to speak freshly from there if they want. No longer will our social patterns be so utterly wasteful of what a person can be. People will always still want many simple restful routines, but freshly newly generated speech and thought will be an understood possibility in our social relations. Concepts like "crossing," “carrying forward," and "unseparated multiplicity” (by these or other names) will play a role in how we understand each other and our contexts.

We can recognize how little the empty categories actually reach, especially in the social realm. Much finer and more effective distinctions can arise directly from the implicit context. For example, government committees are empowered to examine social and economic policies with just the existing categories. Often the members have very limited experiential backgrounds but even when they do there is no room for their individual explications of new aspects that need to be considered. 
[p. 155]

\title{
Appendix: Thinking At the Edge (TAE) Steps
}

\author{
Steps 1- 5 : Speaking from the felt sense
}

Main Instructions

Helpful Details

\section{A felt sense}

Choose something you know and cannot yet say, that wants to be said. Have this knowing as a felt sense (a distinct bodily-felt unclear edge) to which you can always return.

Write it down in a few paragraphs in a very rough way.

From your felt sense, write the central crux in one short sentence, with one key word or phrase, even though the sentence doesn't really say it.

Underline the key word or phrase in the sentence.

Write down one instance.
What you choose to work on needs to be in a field in which you are knowledgeable and experienced. Do not work on a question, but on something that you know. Just a little on from what you are easily able to say, there is something that you know very thickly from years of experience but which is difficult to talk about...it may seem illlogical... marginal... unconventional... awkward... or it may simply be language seems no to work here. If having a felt sense is unfamiliar to you, please consult www.focusing.org.

To find the crux, ask what in this do you wish to articulate? Then, within this, what is the live point for you?

The sentence is just a starting point. It does not need long deliberation. For the moment it states the crux of what you are tracking.

You need a specific example, an event or a time when it actually happened.

\section{More than logical}

Find what does not make the usual logical

What seems illogical may be the most valuable part. Please assure yourself that 
sense and write an illogical sentence.

If you have difficulty writing an illogical sentence, you can write a paradox. you are not dropping this out.

In a paradox something is said to be " $\mathrm{x}$ and also not $\mathrm{x"}$.

\section{No words say what you mean}

Take out the underlined word and write your sentence from Step 1 with a blank slot. Write its usual (dictionary) definition and notice that it is not what you mean.

Return to your felt sense and let another word or phrase come to say what you mean.

Write the usual definition of the second word or phrase.

[p. 156]

Return to your felt sense and let a third word or phrase come.

Write the usual definition of the third word.

Accept the fact that there is no established word or phrase for this knowing.
You recognize, "that's not what I meant". This word would communicate something else. If you are saying something new, none of the words in their usual public meanings will say it exactly.

Make sure it is not just a synonym, but a word with a somewhat different meaning

When you consider its existing public meaning, you see that the second word does not fit either.

[p. 156]

The public meaning of the third word is also not what you meant.

No word fits. None should, if this is new.

\section{What did you want the word to mean? Use fresh phrases}

Although you cannot change the public language, you can write a whole fresh sentence to say what you had wished the single word to mean.

Put the original first word back in the slot in your sentence from Step 1.

Write a phrase or sentence that articulates what you would want the word to mean, what it pulls out from your felt sense which
This time, do not give up your sense. Insist that your sentence does speak from your felt sense. Do not let the word say what it usually says. Wait until you feel this whole sentence speaking from your felt sense, even though most people might not understand it so.

You will need fresh new phrases to say what you would want the word to mean in your sentence. Rather than large public 
the other two do not.

Now put the second word in the slot. Write a phrase or sentence to say what it pulls out from the felt sense.

Do this with the third word.

Write a "string" of all three words and the main fresh phrases in the underlined slot in your sentence from Step 1. At the end of your string add “...”. words, let a new phrase come straight from your felt sense.

Play with the grammar and order. Eliminate excess words until you have a sentence you like. Now you have an elaboration of what you are tracking.

\section{Expanding what you mean, again in fresh phrases}

Using the main words or phrases from Step 4 , write a somewhat odd sentence or two in order to expand even further what you now mean by each of the words or phrases.

In each of the new sentences, underline what is new and important.
Check whether you used any major public words in step 4. If so make fresh phrases to replace those common public words. Let what is new and specific in your felt sense express itself into freshly phrased language. Your sentences might make no sense unless they are understood as you mean them. Here are examples of linguistically unusual sentences: "Knowing the rules is a container from which new ways open". "Definitions stop cellular growth". "Be-having shows something it has". If you let your felt sense speak directly, something linguistically unusual can come.

\section{Steps 6 - 8 Finding Patterns from facets (instances)}

\section{Collecting Facets}

Collect facets, any instances that have actually happened.

Choose three facets and write them with the details which relate to your felt sense. Underline specifics that bring something you
A facet need not illustrate all of your felt sense. A facet can be anything that relates to the felt sense, including times when it came up, what someone said, any incidents even if you cannot tell yourself why they are relevant. Include odd or private things 
might want to keep.

such as "the time the dentist said...". Ask yourself "what has ever

[p. 157]

[p. 157]

Copy your original facet from Step 1 here. Now you have four facets.

happened that has something to do with it?”

General ideas and metaphors are not facets. It isn't an actual event that happened to say "it's like heating something to agitate it".

Any instance is superior to a higher order generalization because it has internal specificity. In any real life event you can discover a complex structure which is actually there.

\section{Each facet may contribute detailed structure}

With each facet:

Notice that there are many intricate relationships between the details. Find a relationship between some details that is relevant to your felt sense.

Restate this relationship in general terms so that it becomes a pattern which can fit many other situations.
In any actual experience there are relationships between details which can give you a new elaboration. Let each facet give you one specific pattern which you did not have before.

Example: The dentist has his thumb in my mouth holding a piece of cotton while he tells me his politics. The pattern is: Speaking to a person who cannot talk back can be intrusive.

\section{Crossing the facets}

You might want to ask: "What does looking from the second facet let me see in the first facet, that I could not see just from within the first facet?"

Write a sentence to capture any new pattern that you want to keep.
You might already have done this.

"Crossing" means attributing the point of one facet to the other. What new aspect of the first facet might become visible if you try to say that it has the same pattern as the second facet?

If the facets do not contain a structure for the whole central thing, this may be found 
by looking at each facet through the other.

\section{Writing freely}

Write freely what you are thinking at this juncture.

This is a free space.

\section{Steps 10 - 14 Building Theory}

One purpose of TAE has now been achieved-- to articulate an implicit knowing and make it communicable. I you wish, you can go on to build a formal, logical theory.

\section{Choosing three terms and linking them}

Choose three words or phrases to be your temporary main terms. Name them "A", "B" and "C ".

Now define $\mathrm{A}$ in terms of $\mathrm{B}$, and also in terms of $C$. First write each equation as an empty formula. “A = B”. “A = C”. Replace the = sign with the word "is." Fill in the words or phrases which A and B and C stand for. Now you have two sentences which might be quite right or quite wrong.

[p. 158]

If necessary modify the sentences. Find the smallest change you can make, so that the "is" becomes true in some respect. Insure that you keep the crux of your felt sense.
A term is not a sentence. For example, "something that moves from the inside" is a term. A sentence always has at least two terms, a subject and a predicate.

Look at your words, phrases and patterns from allof the preceding steps. Make a list of possible candidates for main terms. Choose what feels most important.

Imagine a triangle connecting the three terms. Choose the terms so that most of your territory

\section{[p. 158]}

and your central crux fall within the triangle. Other important ideas can be brought in later at Step 12.

By equating A and B you are "defining” A by using B. Since both terms arose from the same felt sense, there will be a way in which such a connection is true.

If the sentence is grammatical and true and speaks from your felt sense, let it stand. If not, keep the word "is" (or "are") and add or 
change as little as possible so that the assertion is true and speaks from your felt sense. You can insert "is something which". If the sentence seems too inclusive, you can say "some," "one kind,” "is at least," e.g. "one kind of A is B."

Now you have one true sentence that connects $\mathrm{A}$ and $\mathrm{B}$, and one that connects $\mathrm{A}$ and $\mathrm{C}$.

\section{Finding inherent relations between the terms}


Add the word "inherently" after the "is" in each of your two sentences. A is inherently B. A is inherently C. You do not yet know what this will turn out to mean.

Now dip into the intricacy of the felt sense to find out why A is inherently B. Why are these two things inherently connected? What is the very nature of "A", such that it has to be "B"?

Do this also with "A is inherently C."

Write down what you find. Explain the inherent connections. Underline every inherent link you found between A and B and $\mathrm{A}$ and $\mathrm{C}$.
Since "A" and "B" express one felt sense, it will be the case that "A" is inherently "B", not only that it happens to be "B."

This requires entering into the felt sense behind the two terms. Ask "What is A?" "What is B?" You discover some respect in which A is B. There has to be an "Aha." Of course! A always was nothing but the sort of thing that has to be $\mathrm{B}$.

You may get " $\mathrm{A}$ is $\mathrm{X}$, and $\mathrm{X}$ is $\mathrm{Y}$ " and "aha, I see that $\mathrm{B}$ is also Y." So via the fact that they are both $\mathrm{Y}$, they are inherently related to each other. $\mathrm{X}$ and $\mathrm{Y}$ are inherent links between $\mathrm{A}$ and $\mathrm{B}$

\section{Choosing Permanent Terms and Interlocking them.}

Build a new and expanded A term. Choose A, B or C from Step 10. Ask yourself, "What is my central more than logical crux?” Put this whole crux into the A term. One way to do this is by filling in the sentence, " $\mathrm{A}$, which is ... and is...."

The inherent links you found in Step 11 will now be terms. Take the links you found between A and B in Step 11 and write them down.

Now, for your new B, take the inherancy link which is most obviously equivalent to $\mathrm{A}$ and call it B. Then write A is B.
You need not use every inherent link you have, and you may need additional links as you bring in more terms.

When you link any two terms with "is" you may need to use "some," "one kind," or "is something which" in your sentence. for example, "A is something which makes B."

There is an excitement because you can see you are going to be able to derive and define each next thing from your theoretical nucleus.

Once you have a term, keep it the same for every occurrence of that term. The logical power depends on the terms staying the same. Once you have the underlying logical connections they can give power to many differently worded versions, for instance in 
letters, papers, or conversations.

[p. 159]

To develop your theory continue in this way. Take the link closest to $\mathrm{B}$ and call it $\mathrm{C}$ and write $B$ is $C$ and so on. So you have $A=B$; $\mathrm{B}=\mathrm{C} ; \mathrm{C}=\mathrm{D}$ and so on.

You can do the same with the chain of inherent links you found between A and C of Step 10.

A TAE theory is both logical and experiential. The equal sign does not eliminate the different intricacy of each term. That is why equating can be exciting and informative. On the formal logical side the two terms are interchangeable, but on its experiential side the inherancy equation is an understanding. It is not really an equation of two units regardless of content. Moving between the two sides can lead to further terms you may need.

You can now bring up important words or phrases you have not yet used and find the inherent link between the new aspect and one of your terms.

Substitute terms to generate new sentences in the following way: If $\mathrm{D}=\mathrm{A}$ and $\mathrm{A}=\mathrm{B}$, then $D=B$. The sentence $D=B$ is new.

Less formally, you can group what you have not yet used under one or another of the main terms to which it could be equivalent. You can then substitute them in to generate more sentences which logically follow. 
significant.

\section{Applying your theory outside your field}

This is a playful and quick step.

The new pattern in your terms can serve as a "model". Apply the pattern to any large area such as art, religion, education, metaphor.

Write a sentence such as "Education (or some aspect of education) is A". Now wait for something to leap up which makes the sentence true. Write what you find.
How might your pattern allow you to say something about human nature, or society, or the state, groups, interpersonal relations, the physical sciences, truth, beauty, ethics, writing, sexuality, language -- any one large idea?

Or, choose something specific, rather than the whole idea.

We know that the pattern you have articulated can happen in human experience because it did in your facets. The pattern is probably not yet known. These large ideas are unclear accumulations of much meaning and experience.

[p. 160]

Looking at a large idea through your theory may reveal something that is or should be true.

This step is playful unless you happen to be an expert on that topic. Then you could develop it.

\section{Expanding and applying your theory}

This is the serious development of your theory. It may continue for years. 
One way to expand your theory is to ask:

"What is a next question or a new understanding to which it leads?

Add inherent links if necessary so that you can derive what is needed.

After the new term is linked, see by substitution what your other terms are able to say about it.

You can expand your theory further and further in this way.

To apply your theory choose a related area, observation, event which you would like to be able to explain or clarify. Where might your theory make an important difference?

Freshly define this in the terms from your theory..
If you take your theory seriously, what must be supplied immediately before you can consider anything further?

If your theory implies something you don't mean, what further term or distinction would correct it?

Recalling an actual instance may help you formulate the new distinction.

Ask yourself, "How can my novel pattern restructure this?” Look at it through your pattern. Formulate it as an instance of your pattern. If you define it this way, what differences or specific aspects emerge?

What might your theory show that could be valuable for a person working on this topic? What further question would your theory lead one to ask? You are creating new concepts.

Do not let fixed definitions or old ways of thinking limit what you say, even if the topic is large and there is a well established view.about it. Do not hesitate to restructure it. We call such restructuring a "reversal" of the usual way. Something new and specific is easily submerged by the existing assumptions about the larger topic. .

People sometimes believe that their new theory "must be" what some older existing theory "really means", if correctly understood. But the older theory alone does not give people this precise understanding. How does A

The function of a theory is social. Being able to speak precisely from your felt sense builds your understanding into our world. 
The Focusing Institute January, 2003

[p. 161] 


\section{References}

Crease, Robert P. (2004). Philosophy of Science: How Science Moves. The Folio, 19 (1), 1-8.

Gendlin, E.T. (2004). Introduction to 'Thinking at the Edge'. The Folio, 19 (1), 1-8.

Gendlin, E.T. (1997a). A process model. New York: The Focusing Institute.

Gendlin, E.T. (1997b). The responsive order: A new empiricism. Man and World, 30 (3), 383411.

Gendlin, E.T. (1997c). Paper edition. Experiencing and the creation of meaning: A philosophical and psychological approach to the subjective. Evanston, Illinois: Northwestern University Press.

Gendlin, E.T. (1995). Crossing and dipping: some terms for approaching the interface between natural understanding and logical formulation. Minds and Machines 5 (4), 547-560.

Gendlin, E.T. (1992). The primacy of the body, not the primacy of perception. Man and World, 25 (3-4), 341-353.

Gendlin, E.T. (1991). Thinking beyond patterns: body, language and situations. In B. den Ouden \& M. Moen (Eds.), The presence of feeling in thought, pp. 25151. New York: Peter Lang.

All Gendlin articles are also available on www.focusing.org, Gendlin Online Library. 